\title{
An instrument for the simultaneous acquisition of size, shape, and spectral fluorescence data from single aerosol particles.
}

\author{
Edwin Hirst ${ }^{\mathrm{a}}$, Paul H Kaye ${ }^{\mathrm{a}}$, Virginia Foot ${ }^{\mathrm{b}}$, James M Clark ${ }^{\mathrm{b}}$, and Philip B Withers ${ }^{\mathrm{b}}$. \\ ${ }^{\mathrm{a} S}$ Science \& Technology Research Institute, University of Hertfordshire, Hatfield AL10 9AB, UK. \\ ${ }^{\mathrm{b}}$ Defence Science \& Technology Laboratory, Porton Down, Salisbury, Wilts. SP4 0JQ, UK.
}

\begin{abstract}
We describe the construction of a bio-aerosol monitor designed to capture and record intrinsic fluorescence spectra from individual aerosol particles carried in a sample airflow and to simultaneously capture data relating to the spatial distribution of elastically scattered light from each particle. The spectral fluorescence data recorded by this PFAS (Particle Fluorescence and Shape) monitor contains information relating to the particle material content and specifically to possible biological fluorophores. The spatial scattering data from PFAS yields information relating to particle size and shape. The combination of these data can provide a means of aiding the discrimination of bio-aerosols from background or interferent aerosol particles which may have similar fluorescence properties but exhibit shapes and/or sizes not normally associated with biological particles. The radiation used both to excite particle fluorescence and generate the necessary spatially scattered light flux is provided by a novel compact UV fiber laser operating at $266 \mathrm{~nm}$ wavelength. Particles drawn from the ambient environment traverse the laser beam in single file. Intrinsic particle fluorescence in the range $300-570 \mathrm{~nm}$ is collected via an ellipsoidal concentrator into a concave grating spectrometer, the spectral data being recorded using a 16-anode linear array photomultiplier detector. Simultaneously, the spatial radiation pattern scattered by the particle over $5^{\circ}-30^{\circ}$ scattering angle and $360^{\circ}$ of azimuth is recorded using a custom designed 31-pixel radial hybrid photodiode array. Data from up to $\sim 5,000$ particles per second may be acquired for analysis, usually performed by artificial neural network classification.
\end{abstract}

Keywords: Bio-aerosol, detection, spectral fluorescence, spatial light scattering, fibre laser, particle shape, particle classification.

\section{INTRODUCTION}

In a wide variety of environmental, occupational, defence and industrial scenarios, fine particles, typically within the size range from a few tenths of a micrometre to a few hundred micrometres, play an important role. In the defence field, the deliberate generation of hazardous aerosols has posed a major threat since their first substantial use in World War I, and today a wide variety of biological and chemical weapons are believed to be possessed by some national governments and terrorist organisations. The in-situ characterisation of airborne particles has therefore become an important objective in both civilian and military fields, and considerable effort has gone into developing techniques which can analyse particle parameters in real-time and provide some degree of identification or classification.

For example, a potentially powerful technique of airborne particle analysis involves the introduction of individual particles into a near vacuum where they are fragmented using an intense laser light pulse. The resulting atomic and molecular fragments are then measured using a time-of-flight mass spectrometer or similar, yielding a detailed assessment of the material content of the particle (see, for example ${ }^{1}$ ). Such methods offer a high degree of particle discrimination but remain expensive and cumbersome to implement. Furthermore, they are comparatively slow in terms of the rate at which individual particles can be analysed, and since the hazardous aerosol particles of interest may represent a very small percentage of the total aerosol population, they do not currently offer the real-time aerosol analysis capability (ie: response to a change in aerosol composition within a few seconds) desired in monitoring applications. 
Of other possible particle characterisation techniques, those based on elastic light scattering have become popular because they offer genuine real-time non-destructive particle analysis. In their simplest form, optical scattering instruments are designed to draw ambient airborne particles through a measurement space in single file. A light source, usually a laser, illuminates the measurement space and each particle thus scatters some radiation to an appropriately positioned detector. The magnitude of the scattered radiation may, to a first order, be used to determine a particle size, whilst the rate of generation of the light pulses can be related to particle concentration within the sampled atmosphere. Whilst comparatively straightforward to implement, simple light scattering techniques such as these do not yield sufficient information about the particles to provide anything other than a very superficial overview of the ambient aerosol. They do not, for example, provide any indication of the material nature of the particles, whether the particles are of solid or liquid form, or whether the particles are of biological or non-biological origin.

In order to discriminate more effectively between airborne particles of different types, a number of methods have been developed which measure multiple parameters from individual particles. For example, analysis of the spatial distribution of light scattered by each individual airborne particle passing through the measurement space of an optical scattering instrument has proved to be an effective method of improving particle discrimination ${ }^{\text {e.g }}{ }^{9-17}$. This is because the spatial pattern of scattered light contains information relating to the shape of the scattering particle (see Section 2). Using this type of instrument, particles may be effectively classified on the basis of their shape (whether, for example, spherical, cuboidal, flake-like, or fibrous) as well as on their size, the latter being derived from an assessment of the total scattered intensity.

However, spatial light scattering analysis instruments of the type described above cannot discriminate particles on the basis of their material nature. For example, a non-biological silicate based particle may yield an essentially identical spatial scattering pattern to a biological cell of similar size and shape. In order to discriminate particles on the basis of their material structure it is necessary to employ other techniques such as an analysis of light which is scattered inelastically by the particle. Such light is manifest as either fluorescence emission or, far more weakly, Raman emission. Although Raman signals have successfully been recorded from individual levitated particles, the period of illumination required to acquire satisfactory spectra is again too long to allow real-time aerosol monitoring. In contrast, several groups have demonstrated successful measurement of fluorescent spectra from single particles and have used this technique, usually in combination with particle size measurement, to aid particle discrimination on the basis of fluorescence. Some instruments, for example ${ }^{2-4}$, recorded simply an integrated measure of the broadband fluorescence from each particle and used this to attempt discrimination between biological and non-biological particles. Others employed spectrometers to record detailed intrinsic fluorescence spectra from individual particles ${ }^{5-8}$, though not normally at rates commensurate with real-time monitoring applications.

The instrument we describe falls into this category of multi-parameter spectral fluorescence aerosol monitors, though it is unique in acquiring data relating to particle size, shape, and intrinsic fluorescence, thereby offering potential for differentiation between particles having similar size and fluorescence characteristics but differing morphology.

\section{SPATIAL LIGHT SCATTERING}

The manner in which a particle spatially scatters incident light is a complex function of the size, shape, composition, and orientation of the particle, as well as of the properties of the illuminating radiation (wavelength, polarization). With suitable control of some of these variables it is often possible to determine parameters relating to the shape and structure of the scatterer. Fig. 1 shows a typical scattering geometry used to record these spatial scattering patterns; fig. 2 illustrates the wide variety of spatial scattering patterns that can be obtained from individual airborne particles.

Fig 1: Schematic diagram of optical geometry used to acquire spatial scattering profiles from individual airborne particles.

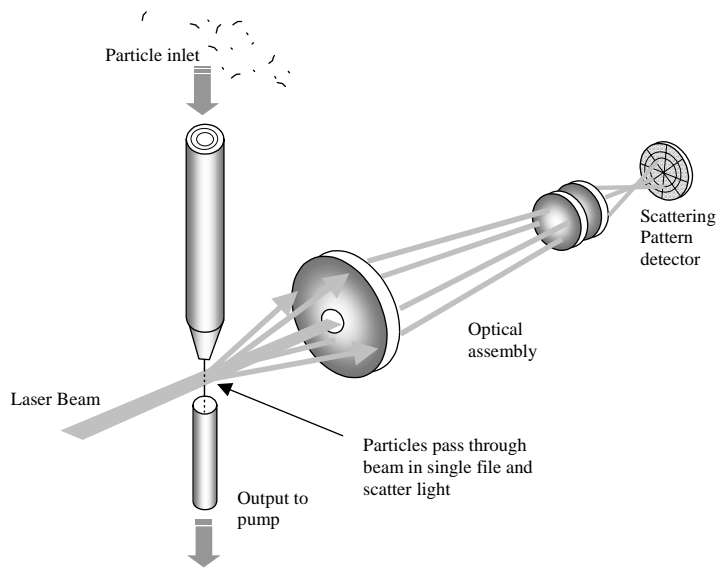



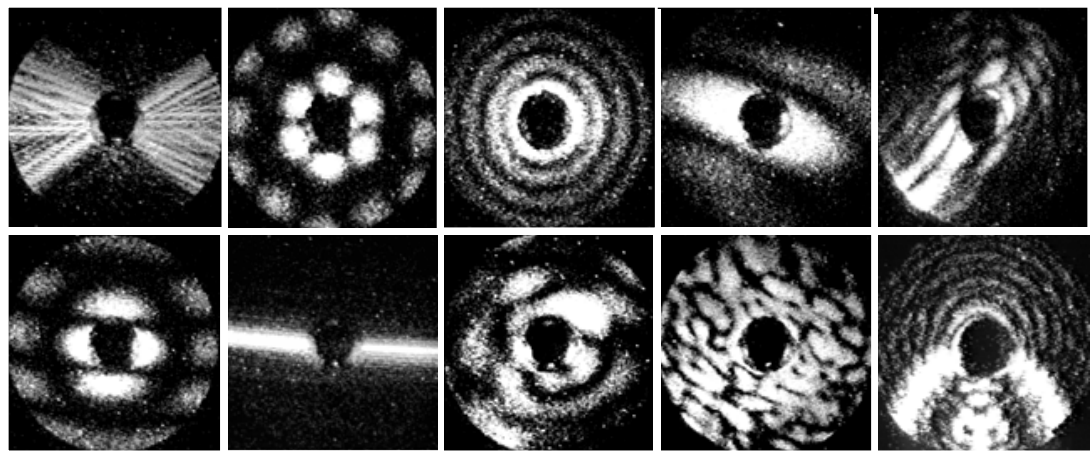

Fig 2: Spatial scattering patterns recorded from individual airborne particles. Top row L-R: curved fibre ( $\sim 5 \mu \mathrm{m}$ length); salt crystal corner-on, $(\sim 3 \mu \mathrm{m})$; water droplet $(9 \mu \mathrm{m})$; haematite ellipsoid $(2 \mu \mathrm{m})$; copper flake ( $\sim \mu \mathrm{m}$ across). Bottom row L-R: salt crystal edge-on $(3 \mu \mathrm{m})$; straight fibre $(\sim 0.4 \mu \mathrm{m}$ thickness); irregular particle with fine surface structure; irregular particle with coarse surface structure; binary droplet. Scattering angle range: $0-30^{\circ}$
The patterns in Fig. 2 were recorded in the laboratory as part of a series of detailed studies into the use of spatial scattering for particle classification ${ }^{9-11}$. They were recorded using an intensified charge-couple device camera (ICCD) capable of single photon detection. The camera was bulky and slow but provided a means of capturing in detail even weak scattering features. In each pattern, the beam direction is perpendicular into the page, the dark central circle being the shadow of the beam-stop. The extreme circumference of the patterns corresponds to $30^{\circ}$ scattering.

The authors have also explored the potential of spatial light scattering analysis for real-time particle shape characterization using discrete azimuthal and/or radial measurements of scattered light to determine a shape index for each particle ${ }^{12,13}$. This approach has resulted in a number of real-time monitoring systems for application in, for example, environmental aerosol monitoring ${ }^{13}$, ambient asbestos detection ${ }^{14}$, and bioaerosol detection ${ }^{18}$. Other workers have similarly employed spatial light scattering analysis, in certain geometries referred to as two-dimensional angular optical scattering (TAOS), for particle characterization. Dick et al. ${ }^{15}$, for example, have used multi-angle azimuthal measurements to determine the spherical and non-spherical fractions of laboratory and atmospheric sub-micrometre aerosols; Holler et al. ${ }^{16}$ used TAOS to examine and classify airborne spore clusters; Sachweh et al ${ }^{17}$ investigated spatial light scattering using three detection geometries and found that not only good particle shape characterization was obtainable but also that, for larger particles, the surface structure of the particle contributed to the scattering pattern provided the size of surface features substantially exceeded the wavelength of illumination.

\section{INSTRUMENTATION DEVELOPMENT}

The instrument described in the remainder of this paper is a development of an earlier real-time aerosol analysis system, MPAM $^{18}$. That instrument employed a 31-pixel hybrid photodiode (HPD) to record particle spatial scattering data and a single photomultiplier detector (PMT) to record intrinsic fluorescence, as shown in Fig.3.

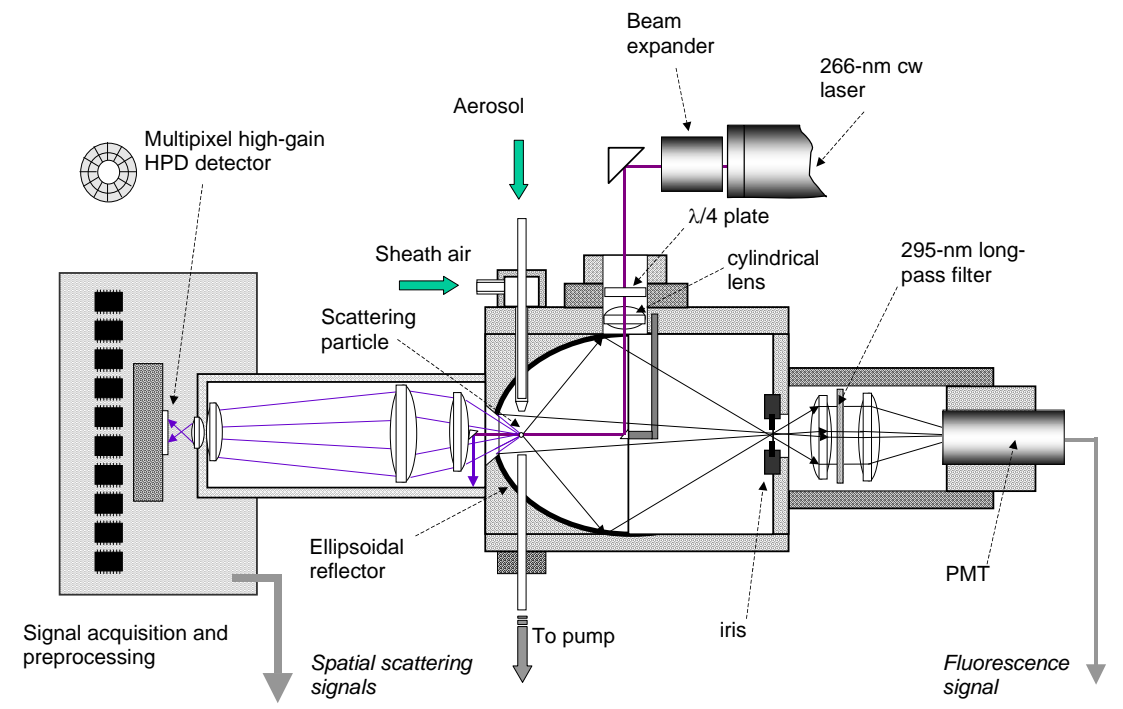

Fig. 3: Schematic of instrument employing spatial scattering analysis and single-channel fluorescence to provide real-time particle characterisation. 
Both excitation of fluorophores and particle spatial scattering were provided by a UV continuous-wave frequency quadrupled Nd-YAG laser, capable of delivering $266 \mathrm{~nm}$ radiation at powers in excess of $100 \mathrm{~mW}$. Despite only a single measurement of broad-band particle fluorescence being recorded, the instrument demonstrated potential for particle classification as illustrated in Fig 4, though the size, power requirements, and fragility of the laser precluded anything other than laboratory use.

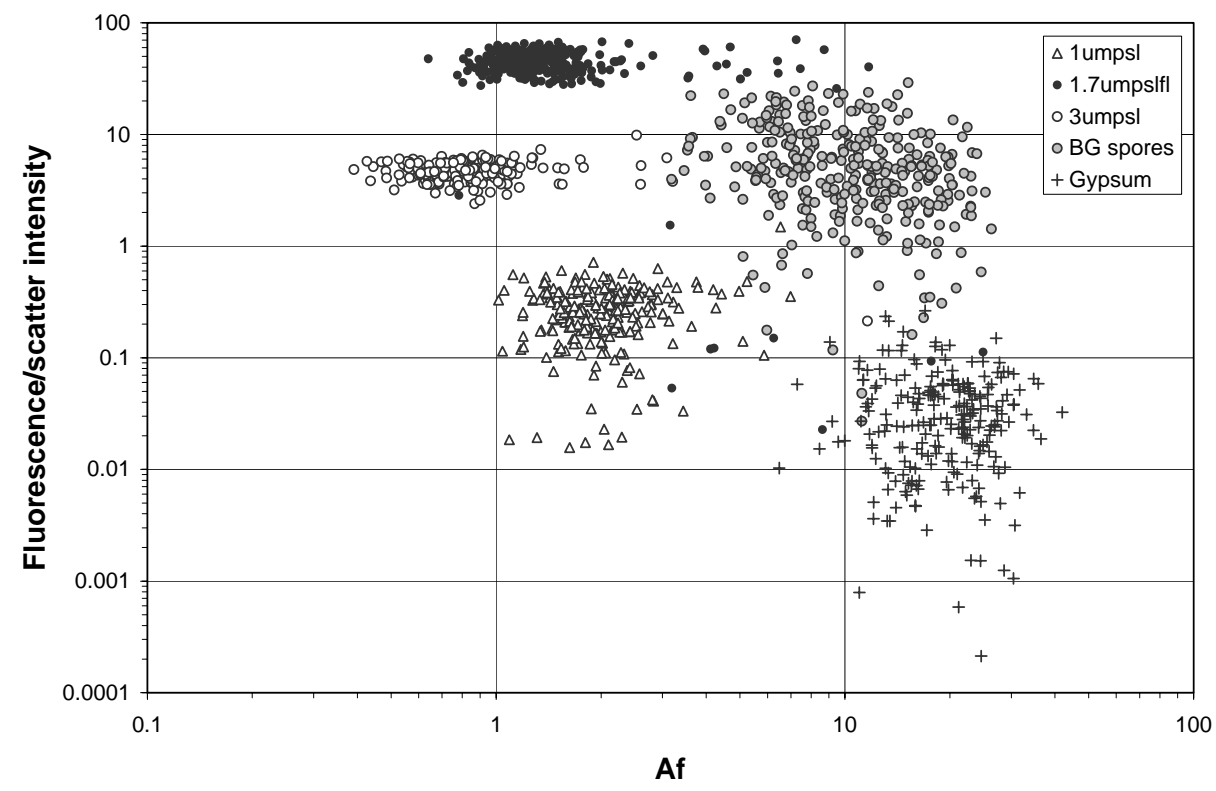

Fig.4: Particle classification data recorded from a variety of aerosol types using the instrument shown in Fig.3.

The graph ordinate in Fig.4 is the ratio of fluorescence intensity measured by the PMT to elastic scatter intensity measured by the HPD detector. The abscissa shows the Asymmetry Factor, Af, a measure of particle shape derived from the root-mean-square variation in azimuthal scattering from the particle scaled such that perfectly spherical particles have an Af $=0$, whilst elongated fibres have an Af approaching 100. The data shown correspond to monodisperse polystyrene latex aerosols (“1umpsl” and “3umpsl”), fluorescently doped psl (“1.7umpsl fl”), BG spores (Bacillus subtilis var. niger), and gypsum dust.

Whilst for these particle types the differentiation achieved by the instrument was good, more realistic ambient aerosols containing more complex and diverse particle types could defeat attempts at particle classification and, specifically, the differentiation of biological particles likely to be pathogenic in nature. Further information relating to the material nature of the particle was necessary to overcome this limitation and particle intrinsic fluorescence spectroscopy was selected for this purpose.

The use of particle intrinsic fluorescence spectroscopy was not new - indeed several instruments had previously been developed to measure the emission spectra of single aerosol particles as indicated in Sec. 1. However, the combination of particle intrinsic fluorescence spectroscopy and particle shape determination (from spatial scattering analysis) offered potential in helping to overcome a common limitation of existing instruments, that of failure to discriminate biological particles from non-biological particles of similar size and fluorescence characteristics. For, example, unburnt fuel droplets were known to cause false positive readings in some particle fluorescence monitors because of the similarity between their fluorescence spectra and that produced by, for example, the bio-fluorophore tryptophan. Discriminating spherical droplets from possibly near-spherical or spheroidal biological particles on the basis of shape would help reduce the occurrence of false-positives. 


\section{PFAS (PARTICLE FLUORESCENCE \& SHAPE) MONITOR}

A schematic diagram of the PFAS monitor currently being commissioned is given in Fig. 5. It comprises a UV fiber laser to provide quasi-continuous illumination of the particle sample flow, a particle scattering/fluorescence chamber, a particle 'shape' analysis system, and a fluorescence spectrometer. Data relating to particle spatial scattering and intrinsic fluorescence are recorded for each particle at rates of up to 5,000 particles/second.

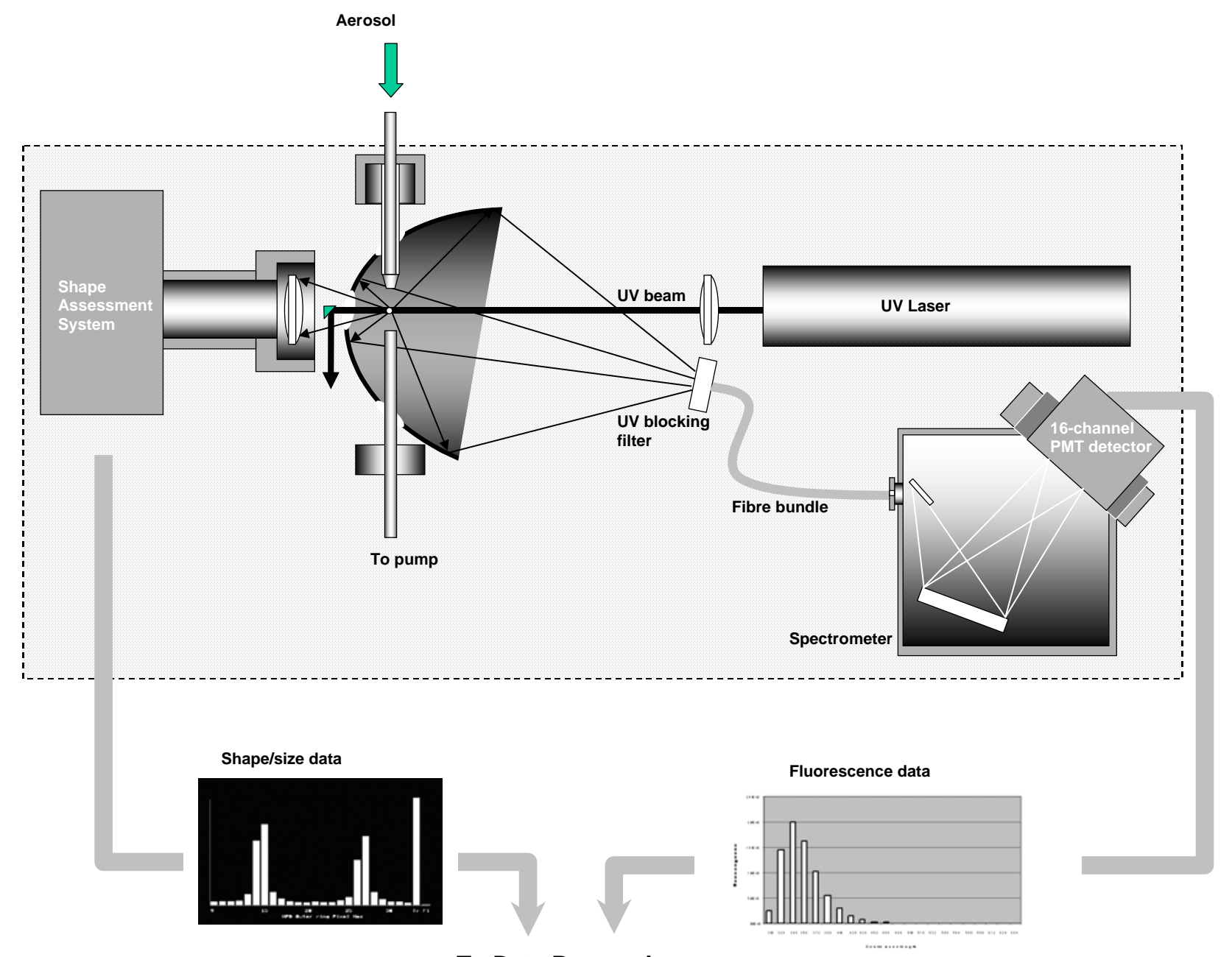

To Data Processing

Fig. 5. Schematic diagram of PFAS monitor.

\subsection{PFAS Operation}

Ambient aerosol is drawn into the instrument at a rate of approximately $5 \mathrm{l} \mathrm{min}^{-1}$. Approximately $80 \%$ of this flow is filtered through a HEPA filter to remove particles greater than $\sim 2 \mu \mathrm{m}$ diameter, and is then recombined with the remaining flow in the form of a sheath. The combined sheath and sample aerosol flows are aerodynamically focused via a tapering delivery nozzle. This has the effect of reducing the sample flow diameter to approximately $0.5 \mathrm{~mm}$, significantly less than the width of the illuminating laser beam (see below), and of causing non-spherical particles to adopt preferential alignment with their longest axis parallel to the flow direction. This latter effect is important in the subsequent interpretation of spatial scattering patterns since the patterns are dependent on both particle shape and orientation, and classification of particle shape is significantly enhanced if orientation is controlled for non-spherical particles. 
Three millimeters below the exit of the delivery nozzle, the sample airflow passes through the beam from a $266 \mathrm{~nm}$ UV fiber laser (see Sec. 4.2), the intersection of the two defining the so-called scattering volume. The beam is optically configured to have an elliptical cross-section at this point of approximately $2.5 \mathrm{~mm}$ diameter and $100 \mu \mathrm{m}$ depth. The near top-hat profile across the beam means that particles contained within the $0.5 \mathrm{~mm}$ diameter sample flow experience similar UV irradiance regardless of their exact trajectory, a requirement for consistent particle size estimation.

Each individual particle passing through the scattering volume results in radiation scattered in the forward direction (up to $30^{\circ}$ from beam axis) being imaged onto a hybrid photodiode (HPD) detector similar to that used in the instrument described in Sec.3. The HPD comprises three annulae, the outermost divided into 24 detector elements, the next divided into 6 elements, and the innermost being a continuous element . The outer two annulae are used in the determination of particle shape, whilst the innermost, being least sensitive to particle orientation is used in the assessment of particle size. The central disc of the detector subtending a scattering angle of $0-5^{\circ}$ is shadowed by the laser beam stop.

Intrinsic fluorescence from the illuminated particle is reflected by an angled ellipsoidal mirror, as shown in Fig.5, via a UV blocking filter at $295 \mathrm{~nm}$ (WG-295, CVI Laser Corp., Albuquerque, NM, USA) into a UV transmitting optical fiber bundle of circular cross-section and $5 \mathrm{~mm}$ diameter. The exit end of this fiber bundle is rectangular in cross-section so as to maximize light delivery into the $8 \mathrm{~mm}$ high entrance slit of a concave grating spectrometer (Jovin Yvon model CP140-1602; 285-715nm grating). At the focal plane of the spectrometer, the resulting spectrum falls onto a 16-anode linear array photomultiplier tube (Hamamatsu R5900U-L16) which covers the $300-570 \mathrm{~nm}$ part of the spectrum. The use of such a photomultiplier for fluorescence spectroscopy has been successfully demonstrated by Pan $e t a l^{19}$ who employed a 32-channel device in the acquisition of transient spectra from particles in flow.

The duration of the signals acquired by the HPD detector and the multi-anode PMT detector is typically 5 5 s, this being the time-of-flight of a particle through the laser beam. For each particle, therefore, the 31 signals from the HPD detector and the 16 signals from the PMT detector are integrated and digitized before being transmitted, together with particle time-of-flight data, to a host computer for analysis and display. Since particles in the flow have very low Reynolds numbers, their times-of-flight should be similar. By defining upper and lower limits of acceptable times-of-flight, occurrences such as particle coincidence in the scattering volume or spurious noise pulses may be identified and earmarked in the subsequent dataset. The overall data acquisition process is rapid enough to allow data from up to $\sim 5,000$ particles $\mathrm{s}^{-1}$ to be recorded - corresponding to all particles in the sampled aerosol for concentrations up to $\sim 10^{3}$ particles $\mathrm{ml}^{-1}$.

\subsection{Fiber laser}

As stated, our previous particle fluorescence spectrometer (Sec.3) employed a cumbersome continuous wave frequency-quadrupled Nd-YAG laser to elicit both spatial scattering and fluorescence data. Other workers ${ }^{\text {eg:3-8 }}$, in an effort to achieve some instrument fieldability, have used more compact externally triggered pulsed solid-state UV lasers to excite particle fluorescence, using the transit of the particle through a separate continuous-wave visible diode laser (or similar) to generate the necessary timing trigger. In the present PFAS monitor, a newly developed optical fiber laser has been employed (Stratophase Ltd., Romsey, UK). Strictly speaking, this is a pulsed laser, though the pulse frequency ( $80 \mathrm{MHz}$ ) means that it may be considered quasi-continuous in respect of the particle illumination, each particle being illuminated by up to 400 pulses. The average beam power at approximately $266 \mathrm{~nm}$ wavelength is variable up to 100 $\mathrm{mW}$ maximum. Being almost as compact as a conventional frequency-quadrupled Nd-YAG laser of similar output power, the fiber laser is small enough to be suitable for use in field equipment, and its efficiency is high enough to ensure that convection cooling is adequate for the laser head.

\subsection{Data Processing}

Initial assessment of the data from the PFAS monitor may be derived from real-time computation each particle's Asymmetry Factor and modal fluorescence magnitude, similar to that employed in Fig.4. However, the Asymmetry Factor represents a crude metric of particle morphology as much potentially useful information contained in the recorded scattering pattern is lost in the processing. A more sophisticated and potentially successful approach is based on the use of artificial neural network (ANN) analysis ${ }^{\text {eg. }}{ }^{14,20}$ of the combined particle scattering and spectral fluorescence data. Classification of the PFAS data by neural network analysis offers the advantage of allowing 
individual instruments to monitor their local environment and 'recognise' transient events which are normal to that environment and not potential threats. These developments will be reported in future communications.

Commissioning of the PFAS monitor is currently underway. Initial particle classification data is anticipated in the early Autumn 2004 and will hopefully be presented at the SPIE conference in London 25-29 October.

\section{ACKNOWLEDGEMENTS}

The work described in this paper has been support by grants from the Defence Science and Technology Laboratory, Porton Down, Salisbury, Wilts. SP40JQ, United Kingdom.

\section{REFERENCES}

1. O. Kievit, A.L. Van Wuijckhuijse, C.E. Kientz, M.A, Stowers, and J.C.M Marijnissen, "Rapid detection of individual biological particles”, J. Aerosol Sci., Proc. European Aerosol Conf., S111-S113, 2004.

2. R.G. Pinnick, S.C. Hill, P. Nachman, J.D. Pendleton, G.L. Fernandez, M.W. Mayo, and J.G. Bruno; "Fluorescent particle counter for detecting airborne bacteria and other biological particles”. Aerosol Sci. Technol. 23, 4, 653664, 1995.

3. P.P. Hairston, J. Ho, and F.R. Quant; "Design of an instrument for real-time detection of bioaerosols using simultaneous measurement of particle aerodynamic size and intrinsic fluorescence”. J. Aerosol Sci. 28, 3, 471-480, 1997.

4. M. Seaver, J. D. Eversole, J. J. Hardgrove, W. K. Cary, Jr., and D. C. Roselle, "Size and fluorescence measurements for field detection of biological aerosols,” Aerosol Sci. Technol. 30, 174-185, 1999.

5. P. Nachman, G. Chen, R. G. Pinnick, S. C. Hill, R. K. Chang, M. W. Mayo and G. L. Fernandez, "Condition sampling spectrograph detection system for fluorescent measurements of individual airborne biological particles”, Appl. Opt. 35, 7, 1069-1076, 1996.

6. G. Chen, P. Nachman, R. G. Pinnick, S. C. Hill, and R. K. Chang, “ Conditional firing aerosol fluorescence spectrum analyser for individual airborne particles with pulsed 266nm laser excitation”, Opt. Lett. 21, 16, 13071309, 1996.

7. Y-L Pan, S. Holler, R. K. Chang, S. C. Hill R. G. Pinnick, S. Niles, and J. R. Bottiger, "Single-shot fluorescence spectra of individual micrometer-sized bioaerosols illuminated by a 351- or 266nm ultraviolet laser”, Opt. Lett. 24, 2, 116-118, 1999.

8. Y-L Pan, J. Hartings, R.G. Pinnick, S.C. Hill, J. Halverson, and R.K. Chang, "Single particle fluorescence spectrometer for ambient aerosols”, Aerosol Sci. Technol. 37, 628-639, 2003.

9. Kaye, P. H., Hirst, E., Clark, J. M., and Micheli. F., Airborne Particle Shape and Size Classification from Spatial Light Scattering Profiles. J. Aerosol Sci.. 23, 6, 597-613, 1992.

10. E. Hirst, P. H. Kaye, and J. R. Guppy, "Light Scattering from Non-spherical Airborne Particles: Experimental and Theoretical Comparisons”. Appl. Opt. 33, 30, 7180-7186, 1994.

11. E. Hirst and P. H. Kaye, "Experimental and Theoretical Light Scattering Profiles from Spherical and Non-spherical Particles”. J. Geophys. Res. (D Atmos.) 101, D14, 19,231-19,235, 1996.

12. Kaye, P.H., Eyles, N.A., Ludlow, I.K. and Clark, J.M. An Instrument for the Classification of Airborne Particles on the Basis of Size, Shape and Count Frequency. Atmos. Environ. 25A, 3/4, 645-654, 1991.

13. P. H. Kaye, K. Alexander-Buckley, E. Hirst, and S. Saunders, “A Real-time Monitoring System for Airborne Particle Shape and Size Analysis”. J. Geophys. Res. (D Atmos.) 101, D14, 19,215-19,221, 1996.

14. E. Hirst, P. H. Kaye and Z. Wang-Thomas, "A Neural Network based Spatial Light Scattering Instrument for Hazardous Airborne Fiber Detection”. Appl. Opt. 36, 24, 6149-6156, 1997.

15. W. D. Dick, P. J. Ziemann, Po-Fu Huang, and P. H. McMurray, "Optical shape fraction measurements of submicrometre laboratory and atmospheric aerosols”, Meas. Sci. and Technol. 9, 183-196 (1998).

16. S. Holler, Yongle Pan, R. K. Chang and J. R. Bottiger, "Two-dimensional angular optical scattering for the characterisation of airborne microparticles”, Opt. Lett. 23, 18, 1489-1491, 1998. 
17. B. Sachweh, H. Barthel, R. Polke, H. Umhauer, and H. Buttner, "Particle shape and structure analysis from the spatial intensity pattern of scattered light using different measuring devices”, J. Aerosol Sci. 30, 10, 1257-1270, 1999.

18. P.H. Kaye, J.E. Barton , E. Hirst, and J.M. Clark; "Simultaneous Light Scattering and Intrinsic Fluorescence Measurement for the Classification of Airborne Particles”. Appl. Opt. 39, 21: 3738-3745, 2000.

19. Y-L Pan, P. Cobler, S. Rhodes, A. Potter, T. Chou, S. Holler, R.K. Chang, R.G. Pinnick and J-P Wolf, "Highspeed, high-sensitivity aerosol fluorescence spectrum detection using a 32-anode photomultiplier tube detector". Rev. Sci. Instrum., 72, 3, 1831-1836, 2001.

20. A.F. Ferguson, T. Sabisch, P.H. Kaye, L.C. Dixon, and H. Bolouri, "High Speed Airborne Particle Monitoring Using Artificial Neural Networks” .Proc. Int. Conference 'Advances in Neural Information Processing Systems 8'. Denver, Colorado, Nov. 1995. MIT Press. Touretzky D.S., Mozer M.C., and Hasselmo M.E., eds. pp. 980-986, 1996. 